\title{
EFFECT OF N-3 POLYUNSATURATED FATTY ACIDS ON LEFT VENTRICULAR FUNCTION IN PATIENTS WITH CHRONIC HEART FAILURE: A META-ANALYSIS OF RANDOMISED PLACEBO-CONTROLLED TRIALS
}

doi:10.1136/heartjnl-2012-302920o.12

Yue-Hua Li, Cheng-Hui Zhou, Xian-Liang Zhou, Ru-Tai Hui, Xian-Liang Zhou.

Cardiovascular Institute and Fuwai Hospital, Chinese Academy of Medical Sciences and Perking Union Medical College

Objectives the objective of this study was to assess the effect of supplementation of n-3 PUFA on left ventricular ejective fraction (LVEF), as a measure of left ventricular systolic function in heart failure patients.

Methods we performed a meta-analysis of randomised, placebocontrolled trials to evaluate the effect of n-3 PUFA on left ventricular function in patients with CHF. Trials were searched in Pubmed, Embase, the Cochrane library database, reviews and reference lists 


\section{ABSTRACTS}

of relevant articles. The weighted mean difference (WMD) was imputed for net changes of LVEF by using random effect models. Meta-regression, sub-group analysis and sensitivity analysis were performed to identify the source of heterogeneity.

Results Six trials (nine comparisons) were included in present meta-analysis. In an overall pooled estimate, compared with placebo group, n-3 PUFA significantly increased LVEF (WMD: $3.57 \%$; 95\% CI 1.57 to $5.41 ; p=0.0004$. Heterogeneity test: $\left.\mathrm{I}^{2}=94 \%, \mathrm{p}<0.00001\right)$. Meta- regression showed that the effect size was not associated with age, gender, dose, duration, proportion of diabetes and atrial fibrillation, New York Heart Association class, baseline LVEF and Jadad score. However, subgroup analysis showed supplementation of n-3 PUFA was more effective with long duration (WMD: 5.06; 95\% CI 1.68 to 8.44; p<0.00001) than short duration (WMD: 0.95 ; 95\% CI 0.07 to $1.81 ; \mathrm{p}=0.22$ ) and in more diabetic heart failure patients (WMD: 2.17 ; $95 \%$ CI 1.39 to 2.95 ; $\mathrm{p}=0.23$ ) than less ones (WMD: 8.46; 95\% CI -5.16 to 22.08; $\mathrm{p}<0.00001$ ).

Conclusions Supplementation of n-3 PUFA may improve left ventricular function in patients with CHF, especially with long duration or in diabetic subjects. 